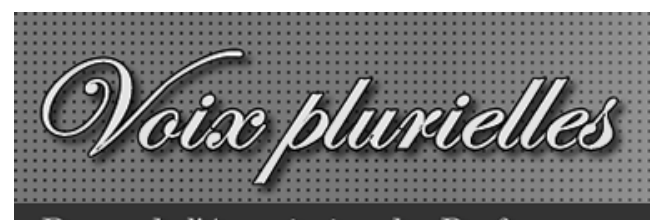

Revue de l'Association des Professeur-e-s de Français des Universités et Collèges Canadiens

\author{
Voix plurielles \\ Volume 3, Numéro 1 : mai 2006
}

Chrystel Morgan

\title{
Le monstre et le transformisme chez Diderot
}

Citation MLA : Morgan, Chrystel. «Le monstre et le transformisme chez Diderot.» Voix plurielles 3.1 (mai 2006).

(c) Voix plurielles, revue électronique de l'APFUCC 2006. 


\title{
Le monstre et le transformisme chez Diderot
}

\author{
Chrystel Morgan \\ Université McMaster
}

Mai 2006

\section{Résumé}

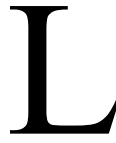

'article suivant se propose d'étudier le transformisme dans les œuvres de Diderot en l'appliquant plus particulièrement à la vue diderotienne du monstre. L'hypothèse que nous proposons est le fait que Diderot pense que le monstre n'est pas en soi « monstrueux » mais que c'est la société qui impose ce libellé aux personnes qui sont « différentes ». Le «monstre », pour Diderot est un produit normal de la nature où tout se transforme. Seule la matière perdure.

Qu'est qu'un monstre? Cette question a hanté l'homme, depuis la nuit des temps. De nombreuses solutions ont été offertes, mais aucune ne s'est écartée de la métaphysique comme l'a faite celle de Diderot. En effet, pour Diderot, la question du monstre peut s'expliquer en termes autres que religieux ou métaphysiques. Pour lui, cette question du monstre est inséparable de la science, de la morale mais aussi de la poétique : « His concern as a scientist was with the nature of monsters, as philosopher with their place in nature and, as a theorist of the arts (both verbal and plastic), with the implications for aesthetics of all that was exceptional in form, whether by accident or design ${ }^{1}$. L'interrogation sur la nature du monstrueux est présente tout au long de l'œuvre de Diderot, mêlée à ses questionnements sur la morale, le matérialisme mais aussi la société et reliée au fait qu'il voit le monstre comme une catégorie de l'histoire naturelle.

En premier lieu, notre analyse va porter sur les monstres physiques que Diderot présente dans Le rêve de d'Alembert. Pour lui, les monstres physiques sont de plusieurs sortes : par excès, par défaut, par renversement et aussi par hybridation ${ }^{2}$. Deuxièmement, nous examinerons le concept du monstre moral que Diderot expose dans deux de ses œuvres, Le Neveu de Rameau et La Religieuse. Enfin, nous nous intéresserons au monstre poétique de Diderot à travers le motif du rêve, qui se retrouve dans deux de ses œuvres, Les Bijoux indiscrets et Le Rêve de d'Alembert. Nous nous baserons sur la classification même que donne Diderot à propos de l'idée de monstre : il transforme le concept de la monstruosité métaphysique en idée rationnelle ${ }^{3}$.

Avant d'entreprendre cette analyse du monstre physique chez Diderot, il faut tout d'abord expliquer ce qu'est pour lui le matérialisme. Il dit que le matérialisme était la seule façon rationnelle de penser l'univers et le monde car il élimine la nécessité d'une présence divine en faveur d'une science absolue, la physique. Dans la conception du monde de Diderot ainsi que de plusieurs de ses contemporains, seule la matière perdure. La matière est dans tout et ne meurt pas, elle ne fait que réarranger le même mécanisme mais sous différentes variations : 
...La matière est éternelle et a en elle la source du mouvement ; le monde est constitué de corpuscules élémentaires incréés, impérissables, inaltérables et indivisibles ; tout l'univers n'est qu'une combinaison des atomes et du vide ; le temps n'est pas plus fini que l'espace ; le monde, ainsi que tout l'univers, est en modification perpétuelle : perpétuelle formation et perpétuelle dissolution; les espèces complexes procèdent des espèces les plus simples et primitivement, la nature a engendré un grand nombre de monstres inaptes à la vie ; l'ordre n'est qu'un cas particulier du désordre, ou d'une autre forme d'ordre ${ }^{4}$.

Pour Diderot, la formation des monstres vient directement de la nature. Le monstre est un phénomène naturel qui découle des fluctuations de cette même nature. D'ailleurs, Diderot définit le monstre comme une variation de la nature, produit des combinaisons infinies qui s'y produisent, puisque les ingrédients de base (temps, matière et mouvement) sont eux-mêmes infinis. Dans Le rêve de d'Alembert, d'Alembert dit, à travers Mlle de Lespinasse : «Qui sait les races d'animaux qui nous ont précédées ? Qui sait les races d'animaux qui succéderont aux nôtres ? Tout change. Tout passe. Il n'y a que le tout qui reste. Le monde commence et finit sans cesse. Il est à chaque instant à son commencement et à sa fin [...] pas une molécule qui ressemble à une molécule ; pas une molécule qui se ressemble à elle-même un instant $\rangle^{5}$. Par conséquent, d'après d'Alembert et le Docteur Bordeu (personnage du texte de Diderot), pour créer un monstre, il suffirait juste de changer une molécule et la composition globale pourrait prendre figure de monstre.

Pour mieux comprendre le matérialisme de Diderot, il faut considérer le fait qu'il l'adopte à cause de son amour de l'ordre et de la science. Pour lui : «The experimental method of science was the only acceptable basis for an interpretation of nature $»^{6}$. À l'époque de Diderot, les monstres sont vus comme des bêtes qui dérangent. Ce sont des créatures dont l'organisation ne s'accorde pas avec le reste de la nature. Le matérialisme permet à Diderot de faire comprendre à ses lecteurs que la nature est aveugle à ses créations, qu'elle ne regarde pas ce qu'elle produit. L'agencement, le flux des molécules produit les êtres et donc aussi les monstres. À partir de cette théorie, on peut comprendre que la création des monstres puisse être infinie. Il peut y avoir autant de monstres qu'il y a de parties du corps humain. En effet, le Docteur Bordeu donne dans Le Rêve de d'Alembert, une leçon d'anatomie ou plutôt un traité sur la formation des monstres ${ }^{7}$. Il explique pourquoi les monstres par défaut et par excès existent. Les monstres par défaut sont ceux auxquels il manque le faisceau d'un des « brins » qui composent leurs organes ou leurs sens et donc, à qui il manque un organe, un membre ou même une capacité. Dans l'exemple du Docteur Bordeu, le faisceau des yeux est affecté ou manquant, il est donc possible qu'alors le « monstre » qui en est issu n'ait qu'un œil au milieu du front, comme le cyclope, ou qu'il soit même aveugle.

Les monstres par excès sont, au contraire, ceux à qui des faisceaux sont en trop. Là aussi, les conséquences sont monstrueuses : un animal à deux têtes, une paire de jumeaux siamois qui partagent les mêmes organes, ou aussi un charpentier dont les organes sont inversés, le coeur à droite au lieu d'être à gauche, exemple de monstre par inversement ${ }^{8}$. Pour Diderot, toute personne peut donner naissance à un monstre. La probabilité de cette naissance est la même que celle de la création d'une erreur dans la nature. Tout est coïncidence. En partant du principe qu'il n'y a 
pas deux molécules identiques, la conception d'un être humain « normal » ou d'un monstre est seulement due au hasard. De plus : " against this background monsters are easily comprehended ; they appear no longer as deviations, but merely as the more eccentric variations in a scheme which depends on no fixed model, only on a flexible bundle of fibers ${ }^{9}$. On peut comprendre alors qu'avec les fluctuations du monde, ceux qui sont des monstres aujourd'hui seront peut-être la norme demain. Le monstre physique de Diderot n'est plus si monstrueux puisque Diderot établit un lien de continuité entre le normal et le monstrueux. Celui-ci peut être soit accidentel soit voulu. C'est là que le docteur Bordeu explique la dernière catégorie de monstres physiques, les hybrides.

Pour Bordeu, si on explore à fond l'idée du transformisme, on en vient à déclarer que : " L'Homme n'est peut-être que le monstre de la femme, et la femme le monstre de l'homme ${ }^{10}$. Ainsi, être normal n'est pas une donnée de la nature, mais devient relatif à l'idée qu'une société se fait de la norme. Bordeu pousse cette conception plus loin quand il parle de créer des êtres hybrides dans le but d'enrichir la société. Diderot : «...has Bordeu considered the rich promise for humanity that is to be found in carefully directed bestiality... $\rangle^{11}$. Quand Bordeu fait un discours sur l'hybridation d'un homme avec une chèvre pour créer des esclaves supérieurs, ce n'est que la conséquence logique de son raisonnement. Toutefois, dans le texte, la question de l'hybridation ne semble pas être aussi importante que la réaction scandalisée de Mlle de Lespinasse qui s’écrie : " Vous êtes monstrueux ! ${ }^{12}$. Pour se défendre, Bordeu lui explique que cela ne vient pas de lui mais de la société qui définit et désigne le monstre ${ }^{13}$. Dans sa défense, Bordeu aborde le sujet du monstre moral qui va être analysé dans la seconde partie.

En effet, pour Diderot, la nature est suprême. Quand il naît, l'homme est bon, vertueux et sensible $^{14}$ et donc, ne peut pas être un monstre. Par rapport à cela, Le Neveu de Rameau semble poser problème car le Neveu ne suit apparemment pas cette logique. Dans ce texte, Diderot met en parallèle deux concepts : l'influence de la société sur Lui (le Neveu) mais aussi celle de la nature qui l'a formé et qui détermine la façon dont la société va l'influencer. En effet : « Rameau seems to typify the human monster, the advocate of evil ; he is the disquieting exception to Diderot's rule of human nature ${ }^{15}$. Ce qu'on entend par là est le fait qu'à plusieurs reprises dans le livre, Rameau explique qu'il aime être mauvais et immoral ${ }^{16}$. Cela discrédite donc la théorie de Diderot, que l'homme n'est heureux que dans la vertu. Mais, même si le neveu est immoral par nature, cela veut juste dire que comme le monstre physique, il incarne une variation de la «norme » humaine. De plus, Diderot le voit comme un être composite, comme privé d'une fibre. Dans Le Neveu de Rameau, il dit : «C'est apparemment qu'il y a pour les uns un sens que je n'ai pas; une fibre qui ne m'a point été donnée, une fibre lâche qu'on a beau pincer et qui ne vibre pas; ou peut-être c'est que j'ai toujours vécu avec de bons musiciens et de méchantes gens ; d'où il est arrivé que mon oreille est devenue très fine, et que mon cœur est devenu sourd» ${ }^{17}$. Il est donc clair que pour Rameau lui-même, la nature en tant que telle n'est pas forcément responsable de son manque de moralité, mais que la société l'a affecté de façon négative. Pour Diderot : « Le vrai responsable du crime est le corps social, qui n'est pas assez bien organisé pour que l'énergie de l'homme criminel puisse s'employer dans l'intérêt de tous et non à leur détriment ${ }^{18}$. Donc, pour lui, le monstre moral bien que créé par la nature, est en fait le vrai produit de la société.

Cette idée de la responsabilité de la société dans le développement possible des monstres est encore plus frappante dans La Religieuse que dans Le Neveu de Rameau. En effet, pour Diderot, 
la religion est une offense à la nature humaine ${ }^{19}$, elle fait partie des mœurs ou normes de la société qui vont à l'encontre de la nature. C'est ce conflit entre la nature humaine et les règles de la société qui crée le monstre moral. Comme le Neveu, les religieuses de Diderot sont des monstres, une mystique, une sadique et une lesbienne ${ }^{20}$. En effet, il veut prouver que la répression dont les religieuses font l'expérience est la cause de leur dépravation, de leur monstruosité. Il en vient même à dire que le mal de la nature humaine vient de la répression et des lois imposées à l'homme par la religion et la société pendant si longtemps qu'elles n'ont plus comme but les besoins de l'homme ou même le respect de sa véritable nature ${ }^{21}$. Il est évident que même dans La Religieuse, les monstres sont un produit de la société. Pour Diderot, la moralité édictée par la société ne fait que créer des monstres car elle va contre la nature en créant de fausses normes. Tout comme pour les monstres physiques, ces normes devraient évoluer avec l'homme et la nature, mais elles ne le font pas. Dans La Religieuse, Diderot lance un avertissement à la société : " Qu'on est injuste dans le monde! On permet à un enfant de disposer de sa liberté à un âge où il ne lui est pas permis de disposer d'un écu. Tuez plutôt votre fille que de l'emprisonner dans un cloître malgré elle ; oui, tuez-la. $»^{22}$. Le monstre moral par conséquent, n'est pas un phénomène naturel mais un être qui a été dépravé par la société. Pour Diderot, la culture est à blâmer pour la formation du monstre moral, alors que la nature n'a fait que créer ces personnes aveuglément. La société a créé le monstre moral en allant contre les lois de la nature, produisant un être hybride qui résulte des effets combinés de la nature et de la société.

Diderot démontre cette idée dans la construction même de ses œuvres. En effet la poétique est pour Diderot un moyen sûr de démontrer ses idées. Pour ce faire, il utilise le motif du rêve à plusieurs reprises dans Les Bijoux Indiscrets et bien sûr dans Le Rêve de d'Alembert. Cette troisième partie va analyser cet usage du rêve et démontrer que l'œuvre de Diderot est en ellemême " monstrueuse », hybride et voulue ainsi. Dans Les Bijoux Indiscrets, une œuvre aux apparences assez légères, Diderot utilise le rêve pour communiquer des idées plus sérieuses, qui font partie de son «système de nature ${ }^{23}$. En effet, après de denses conversations métaphysiques, Mangogul va se coucher et rêve. En présentant ce chapitre du texte, Diderot l'introduit comme « Le meilleur peut-être, et le moins lu de cette histoire, Le rêve de Mangogul, ou Voyage dans la région des hypothèses $\gg{ }^{24}$. Il est donc clair que même pour Diderot, cette portion du texte est la plus importante. Le rêve est utilisé comme artifice pour présenter des questions qui peuvent paraître ridicules ou même monstrueuses. Diderot dit lui-même : « ... il y a quelque adresse à avoir mis mes idées dans la bouche d'un homme qui rêve : il faut souvent donner à la sagesse l'air de la folie afin de lui procurer ses entrées $»^{25}$. Cette phrase peut être une référence soit à Mangogul, soit à d'Alembert. Dans chacun de ces cas, Diderot utilise le rêve pour exhiber son matérialisme, pour transcender la métaphysique qui a atteint ses limites.

Pour comprendre la monstruosité poétique de l'œuvre de Diderot, il faut d'abord établir une définition du rêve selon lui. Pour Diderot, le rêve : «... signifies a mode of thinking that is seemingly or superficially absurd, but, on a deeper plane, is just the opposite and expresses a superior intuition or an ingenious supposition $»^{26}$. Le rêve est comme le monstre. Si on le juge par rapport aux normes sociales, il apparaît comme absurde, comme une faute. Mais, si on le contemple comme une variation de la nature, alors, il apparaît comme un autre niveau de la conscience humaine dans lequel aucune inhibition n'est présente et où toute pensée est permise. C'est dans le rêve que les 
lois de la nature sont appliquées sans être contraintes par les lois de la société. Dans le Rêve de d'Alembert, Diderot va plus loin que dans les Bijoux indiscrets, car tout son discours est basé sur le rêve de d'Alembert. Non seulement le rêve est une forme hybride de pensée, mais Diderot pousse l'hybridation jusque dans sa rhétorique. En effet, son matérialisme se retrouve dans le texte :

La structure chiasmique affecte la forme globale de l'œuvre, en ce sens que le dialogue se développe suivant deux axes assimilables aux diagonales de l'art baroque : l'un descend de la région des rêves dans le domaine de la réalité ; sur l'autre ligne, la plus sinueuse et plus évanescente que la première, l'idée ou le personnage remonte de la sphère des réalités à celle des rêves ${ }^{27}$.

Diderot introduit le monstre dans la structure baroque de son œuvre. Dans la mesure où le baroque se définit comme l'art de l'excès, de la surcharge, de la circonvolution, de l'étrange, il se prête au monstrueux. Le texte et son contenu se rencontrent dans ce monde étrange du rêve, ce qui permet à Diderot d'explorer le lien entre la forme et le contenu, ce dernier étant presque sorti d'un conte de fée : «Des intentions caricaturales, des trouvailles grotesques ou fantastiques s'insinuent dans les propos des personnages [...] La structure d'ensemble du dialogue ne fait que renforcer le sens de l'aventure nocturne de d'Alembert $\gg{ }^{28}$. Une seule conclusion est possible : Diderot a voulu créer un monstre poétique. Il a intentionnellement donné cette forme à son œuvre pour pouvoir présenter des discours plus ou moins controversés au travers d'un moyen qui est déjà considéré comme étrange. Son œuvre biscornue ouvre à la psyché humaine des possibilités d'expression qui échappent à la censure de la religion ou de la société et constituent un espace de liberté créatrice et conceptuelle.

En conclusion, pour Diderot, la nature est primordiale pour comprendre l'individu. Dans ses manifestations et ses idées, l'homme n'est rien sans le tout que forme la nature. Le monstre n'existe pas comme tel, mais seulement en fonction des lois de la société et non dans les lois de la nature qui sont inclusives et admettent le continuum et le flux. Il est seulement un élément dans la chaîne du transformisme et du matérialisme de Diderot. Il est possible que le monstre d'aujourd'hui soit la norme de demain. Dans une nature où tout change et où rien n'est constant, seul le tout reste. Comme dans la morale et la poétique, seul le tout est important. Pour Diderot : « ...la vie de l'individu n'a de sens et ses actions de valeur que subordonnées à un dessein général de la nature $»^{29}$, et non assujettie aux normes de la société.

\section{Bibliographie}

Sources Premières

Diderot, Denis. Les Bijoux Indiscrets. Édition de Jacques Rustin. Éditions Gallimard, Folio Classique, 1981.

Diderot, Denis. Le Neveu de Rameau. Paris : GF Flammarion, 1983.

Diderot, Denis. La Religieuse. Paris : GF Flammarion, 1968.

Diderot, Denis. Le Rêve de d'Alembert. Paris : GF Flammarion, 2002. 


\section{$\underline{\text { Sources Secondaires }}$}

Belaval, Yvon. L’Esthétique Sans Paradoxe de Diderot. Bibliothèque Des Idées, Librairie Gallimard, 1950.

Daniel, George. « Autour du Rêve de d'Alembert : Réflexions sur l'esthétique de Diderot. » dans Diderot Studies XII, Otis Fellows et Diana Guiragossian (éds.). Genève : Librairie Droz S.A., 1969.

Hill, Emita. "Human Nature and the Moral Monstre." Dans Diderot Studies XVI, Otis Fellows et Diana Guiragossian (éds.). Genève: Librairie Droz S.A., 1973.

Hill, Emita. "Materialism and Monsters in Le Rêve de d'Alembert." Dans Diderot Studies X, Otis Fellows et Diana Guiragossian (éds.). Genève: Librairie Droz S.A., 1968.

Laidlaw, Norman G. "Diderot's Teratology." Dans Diderot Studies IV, Otis Fellows et Diana Guiragossian (éds.). Genève: Librairie Droz S.A., 1963.

Stenger, Gerhardt. « Nature et liberté chez Diderot après 1'Encyclopédie. » Paris : Universitas, 1994.

Vartanian, Aram. "Diderot and the Phenomenology of the Dream.” Dans Diderot Studies VIII, Otis Fellows et Diana Guiragossian (éds.). Genève: Librairie Droz S.A., 1966.

\section{NOTES :}

${ }^{1}$ Norman G. Laidlaw, “Diderot’s Teratology,” Diderot Studies IV (1963): 109.

${ }^{2}$ George Daniel, « Autour du Rêve de d'Alembert : Réflexions sur l'esthétique de Diderot, » Diderot Studies XII (1969) : 33 .

${ }^{3}$ Daniel 38.

${ }^{4}$ Daniel 60.

${ }^{5}$ Denis Diderot, Le Rêve de d'Alembert (Paris : GF Flammarion, 2002) 94.

${ }^{6}$ Emita Hill, "Materialism and Monsters in Le Rêve de d'Alembert," Diderot Studies X (1968): 69.

${ }^{7}$ Denis Diderot, Le Rêve de d'Alembert (Paris : GF Flammarion, 2002) 117-119.

${ }^{8}$ Diderot 119-120.

${ }^{9}$ Emita Hill, "Materialism and Monsters in Le Rêve de d'Alembert," Diderot Studies X (1968): 82-83.

${ }^{10}$ Denis Diderot, Le Rêve de d'Alembert (Paris : GF Flammarion, 2002) 121.

${ }^{11}$ Norman G. Laidlaw, “Diderot's Teratology,” Diderot Studies IV (1963): 121.

${ }^{12}$ Denis Diderot, Le Rêve de d'Alembert (Paris : GF Flammarion, 2002) 176.

${ }^{13}$ Diderot 177.

${ }^{14}$ Gerhardt Stenger, Nature et liberté chez Diderot après l'Encyclopédie (Paris : Universitas, 1994) 243.

${ }^{15}$ Emita Hill, "Human Nature and the Moral Monstre," Diderot Studies XVI (1973): 101.

${ }^{16}$ Denis Diderot, Le Neveu de Rameau (Paris : GF Flammarion, 1983) 78-79.

${ }^{17}$ Diderot 116.

${ }^{18}$ Gerhardt Stenger, Nature et liberté chez Diderot après l'Encyclopédie, (Paris : Universitas, 1994) 269.

${ }^{19}$ Yvon Belaval, L'Esthétique Sans Paradoxe de Diderot (Librairie Gallimard, 1950) 70.

${ }^{20}$ Emita Hill, "Human Nature and the Moral Monstre," Diderot Studies XVI (1973): 91.

${ }^{21}$ Hill 95.

22 Denis Diderot, La Religieuse (Paris : GF Flammarion, 1968) 104.

${ }^{23}$ Aram Vartanian, "Diderot and the Phenomenology of the Dream," Diderot Studies VIII (1966): 221. 
${ }^{24}$ Denis Diderot, Les Bijoux Indiscrets (Éditions Gallimard, 1981) 171.

${ }^{25}$ George Daniel, « Autour du Rêve de d'Alembert : Réflexions sur l'esthétique de Diderot, » Diderot Studies XII (1969) : 15.

${ }^{26}$ Aram Vartanian, "Diderot and the Phenomenology of the Dream," Diderot Studies VIII (1966): 226.

${ }^{27}$ Ibid.

${ }^{28}$ Ibid.

${ }^{29}$ Ibid. 